\title{
Examining the Neural Correlates of Error Awareness in a Large fMRI Study
}

2 Gezelle Dali ${ }^{1}$, Méadhbh Brosnan ${ }^{2,3,4}$, Jeggan Tiego ${ }^{4}$, Beth P. Johnson ${ }^{4}$, Alex Fornito ${ }^{4}$, Mark

3 A. Bellgrove ${ }^{4 *}$, Robert Hester ${ }^{*}$

$4 *$ These authors contributed equally and should be considered joint senior authors.

5

6 Brief running title

7 Error Awareness Neural Correlates

8

9 Affiliations

$10{ }^{1}$ Melbourne School of Psychological Sciences, The University of Melbourne, 3010

112 Department of Experimental Psychology, University of Oxford, Oxford, UK

$12{ }^{3}$ Oxford Centre for Human Brain Activity, Wellcome Centre for Integrative Neuroimaging,

13 University of Oxford, Oxford, UK

$14{ }^{4}$ Turner Institute for Brain and Mental Health and School of Psychological Sciences, and

15 Monash Biomedical Imaging, Monash University, 3800

16

17 Correspondence

18 Gezelle Dali

19 Melbourne School of Psychological Sciences, The University of Melbourne, Parkville, 3010,

20 Vic., Australia

21 Email: gezelledali@gmail.com 


\section{Abstract}

25 Goal-directed behaviour is dependent upon the ability to detect errors and implement

26 appropriate post-error adjustments. Accordingly, several studies have explored the neural

27 activity underlying error-monitoring processes, identifying the insula cortex as crucial for

28 error awareness and reporting mixed findings with respect to the anterior cingulate cortex.

29 Variable patterns of activation have previously been attributed to insufficient statistical

30 power. We therefore sought to clarify the neural correlates of error awareness in a large

31 event-related functional magnetic resonance imaging (MRI) study. Four hundred and two

32 healthy participants undertook the Error Awareness Task, a motor Go/No-Go response

33 inhibition paradigm in which participants were required to indicate their awareness of

34 commission errors. Compared to unaware errors, aware errors were accompanied by

35 significantly greater activity in a network of regions including the insula cortex,

36 supramarginal gyrus, and midline structures such as the anterior cingulate cortex and

37 supplementary motor area. Error awareness activity was related to indices of task

38 performance and dimensional measures of psychopathology in select regions including the

39 insula, supramarginal gyrus and supplementary motor area. Taken together, we identified a

40 robust and reliable neural network associated with error awareness.

41 Keywords: anterior cingulate cortex, error awareness, error-monitoring processes,

42 functional magnetic resonance imaging, insula 


\section{Introduction}

Error processing facilitates goal-directed behaviour through error detection and the

execution of appropriate post-error adjustments. Within error processing, it is possible to

47 delineate between errors made with and without conscious recognition. Although error

48 processing can proceed in the absence of awareness, conscious perception of errors may

49 subserve the implementation of remedial behaviours. Critically, deficient error awareness has

been associated with symptoms of inattention, lack of insight and perseverative behaviour in

several clinical conditions such as attention-deficit hyperactivity disorder (ADHD; O'Connell

et al. 2009), autism spectrum disorder (ASD; Klein et al. 2013b) and substance use disorder

(Hester et al, 2009), providing impetus for investigating the underlying neurobiology of error awareness.

In performance monitoring tasks, errors are largely associated with an event-related potential (ERP) signature comprising the error-related negativity (ERN) and the error positivity (Pe). The ERN is a negative deflection with a fronto-central distribution that appears 50-100ms following an error (Gehring et al. 1993), whereas the Pe is a positive deflection with an approximate latency of 300-500ms occurring over a centro-parietal location (Falkenstein et al. 1991). Neuroimaging and source localisation studies have identified the anterior cingulate cortex (ACC) as the source of the ERN (Debener et al. 2005; van Veen and Carter 2006). Indeed, the ACC is consistently implicated in performance monitoring tasks and is suggested to navigate the selection and evaluation of goal-directed

64 behaviours (Holroyd and Yeung 2012). The source of the Pe, however, remains somewhat equivocal, with reports that it arises from activity in the rostral ACC (rACC; Herrmann et al. 2004; Van Boxtel et al. 2005), prefrontal (Masina et al. 2019) and parietal cortices (van Veen and Carter 2006; O'Connell et al. 2007). 
With regard to error awareness, most electrophysiological studies argue that the ERN is unaffected by conscious error perception. This pattern has been observed in a myriad of tasks, including anti-saccade tasks (Nieuwenhuis et al. 2001; Endrass et al. 2007), Go/No-Go error awareness tasks (O'Connell et al. 2007; Dhar et al. 2011) and visual discrimination tasks (Steinhauser and Yeung 2010; Endrass et al. 2012). Contrastingly, the Pe has been found to be selectively modulated by error awareness such that greater amplitudes are observed following aware errors (Nieuwenhuis et al. 2001; O'Connell et al. 2007; Steinhauser and Yeung 2010; Dhar et al. 2011; Hoffmann and Beste 2015). There are, however, studies which have demonstrated that both the ERN and Pe are sensitive to error awareness (Scheffers and Coles 2000; Maier et al. 2008; Hewig et al. 2011; Wessel et al. 2011; Shalgi and Deouell 2012). Given such findings, the ERN has been proposed to be the foremost indication that an error has occurred and may serve as a feedforward input signal into systems that are more responsible for error awareness (Murphy et al. 2012; Wessel 2012), whereas the Pe reflects the accumulation of information that leads to error awareness (Klein et al. 2013b). electrophysiological findings. Consistent with findings on the Pe, a network of frontal and parietal regions has been implicated in error awareness, namely the bilateral inferior parietal and bilateral mid-frontal cortices (Hester et al. 2005; Harsay et al. 2012; Orr and Hester 2012). The insula cortex - largely the anterior insula cortex (AIC) - is also widely recognised to be selectively modulated by error awareness (Klein et al. 2013b). While the insula is unlikely to generate the Pe directly, it has been suggested to indirectly elicit the Pe through its functional connections with frontal and parietal cortices (Klein et al. 2007). Corroborating

90 findings on the ERN, the relationship between awareness and the ACC remains a topic of 91 contention. Several earlier studies that have found ACC activity to be greater for errors than correct responses have discerned no difference in activity between aware and unaware errors 
93 (e.g., Hester et al. 2005; Klein et al. 2007). In contrast to the majority of earlier studies,

94 recent investigations have reported dorsal ACC (dACC) sensitivity to error awareness, with

95 increased activity observed during aware errors (Harsay et al. 2012; Harsay et al. 2018).

Although heterogeneity in imaging modalities, sample characteristics and study

97

designs may contribute to discordant neuroimaging findings, they are unlikely to explain

variation observed across several error awareness studies (Wessel 2012). Instead, disparities

in distinguishing ACC activity patterns between aware and unaware errors may be attributed

to inadequate statistical power associated with small sample sizes. For example, we have

previously found no difference in ACC activity between aware and unaware errors in samples

of 13 (Hester et al. 2005) and 16 (Hester et al. 2009a) participants, however have found greater dACC activity for aware errors in a sample of 27 participants (Hester et al. 2012).

Importantly, when the samples of these three studies were collated, a significant effect of power thus seems a robust explanation for these mixed neuroimaging findings (Button et al.

107 2013; Poldrack et al. 2017). Indeed, low power is a pertinent problem for task-based neuroimaging studies where there are often a small number of observations and few participants (Cremers et al. 2017; Turner et al. 2018). Although recent work has begun to address the reproducibility of brain imaging (Bossier et al. 2020), relatively few functional

111 imaging replication studies have been conducted in this area of research. In light of this

112 shortcoming, the neural correlates of error awareness and the influence of measures of task

113 and individual differences warrants further examination.

114 Here, we set out to confirm previous investigations using the motor Go/No Error

115 Awareness Task (Hester et al. 2005), in a large, community-based sample. Behavioural

116 performance on the error awareness task and corresponding event-related neuroimaging were

117 used to assess the neural mechanisms associated with error awareness. Based on the reviewed 
118 literature, we hypothesise that aware errors will be accompanied by greater activity in a

119 network of regions including the insula, parietal and mid-frontal cortices, and midline

120 structures such as the ACC. Further, we extend upon previous investigations by exploring

121 whether variance in awareness-related neural activity is accounted for by measures of

122 individual differences in dimensional measures of psychopathology including ADHD, ASD,

123 and impulsivity.

Materials and Methods

\section{Participants}

Participants were recruited via Monash University Clayton campus, social media and newspaper advertisements along with experimenter networks. All participants were righthanded and had normal or corrected-to-normal vision. Participants were excluded if they were colour blind or reported any history of neurological or psychiatric illness, including head injury, previous usage of psychotropic medication or substance use disorder. All

131 participants provided written informed consent and were reimbursed for participation. The

132 study received approval by the Monash University Human Research Ethics Committee for

133 meeting the research standards prescribed by the National Health and Medical Research

134 Council (CF12/3072 - 2012001562).

Four hundred and seventy-three participants completed the event-related fMRI

136 protocol. Participants were subsequently excluded due to missing functional runs $(n=4)$ or

137 behavioural data $(n=22)$, no signalling of aware errors $(n=37)$, corrupted functional data $(n$

$138=6)$, or distorted anatomical data $(n=2)$. The final sample with complete behavioural and

139 neuroimaging data comprised 402 participants (female, $54.22 \%$; $M_{\text {age }}=23.64$ years, $S D=$

1405.45 ; age range: 18-50 years). Of those, 20 participants (female, 50\%; $M_{\text {age }}=25.55$ years, $S D$

$141=7.25)$ did not have questionnaire data available. Further information on participant age can

142 be found in Table 1A of the Supplementary Material. 
143

144

145

146

147

\section{Experimental Design}

Participants were administered a battery of self-report measures designed to assess a comprehensive range of psychopathological characteristics. The battery comprised the Barratt Impulsiveness Scale, Version 11 (BIS-11; Barratt and Patton 1983) to assess impulsivity, Conners’ Adult ADHD Rating Scales - Self Report: Long Version (CAARS S:L; Conners 1998) to assess ADHD-like behaviours, the Behavioural Inhibition/Activation Systems Scale (BIS/BAS; Carver and White 1994) to measure sensitivity to avoidance and approach motivation, the Autism-Spectrum Quotient (AQ; Baron-Cohen et al. 2001) to assess autistic traits, and the Hospital Anxiety and Depression Scale (HADS; Zigmond and Snaith 1983) to assess anxiety and depression traits.

Error Awareness Task. The error awareness task (see Figure 1) is a Go/No-Go motor inhibition task that presents a serial stream of colour words in a congruent or incongruent colour. Previously, we employed an awareness task with two competing inhibition rules (a repeat No-Go rule and colour No-Go rule). To address concerns that introducing two No-Go rules potentially contaminates the BOLD signal, we opted to remove the repeat No-Go rule. Pilot testing confirmed that unaware error rates with a single No-Go rule were consistent with our previous work (see Table 2A of the Supplementary Material for a summary of pilot data). Thus, participants were required to respond to the incongruent trials (Go trials) with a left button press, while withholding their response when the word and colour were congruent (No-Go trials). To indicate error awareness, participants were trained to forego making a standard 'Go' response and instead execute a right button press following any commission error. Erroneous No-Go trials were those in which a participant failed to withhold a response. To classify erroneous trials for data analysis, unaware errors were those in which the participant responded with a left button press on the No-Go trial and again on 
167 the following Go trial. Any deviation from this pattern of response on or following a No-Go

168 error was classified as an aware error (Figure 1).

169 The task comprised six blocks, each with 175 trials. Across all blocks, participants

170 were administered 900 Go trials and 150 No-Go trials. All stimuli were presented for $900 \mathrm{~ms}$

171 followed by a $600 \mathrm{~ms}$ inter-stimulus interval. An event-related design was employed,

172 distributing the No-Go trials pseudo-randomly throughout the serial presentation of Go trials.

173 Events of interest were adequately separated in order to analyse correct and failed response

174 inhibition events separately without cross-contamination. The number of Go trials separating

175 No-Go trials ranged between 1 and $12(M=6.23 ; S D=2.55)$. 


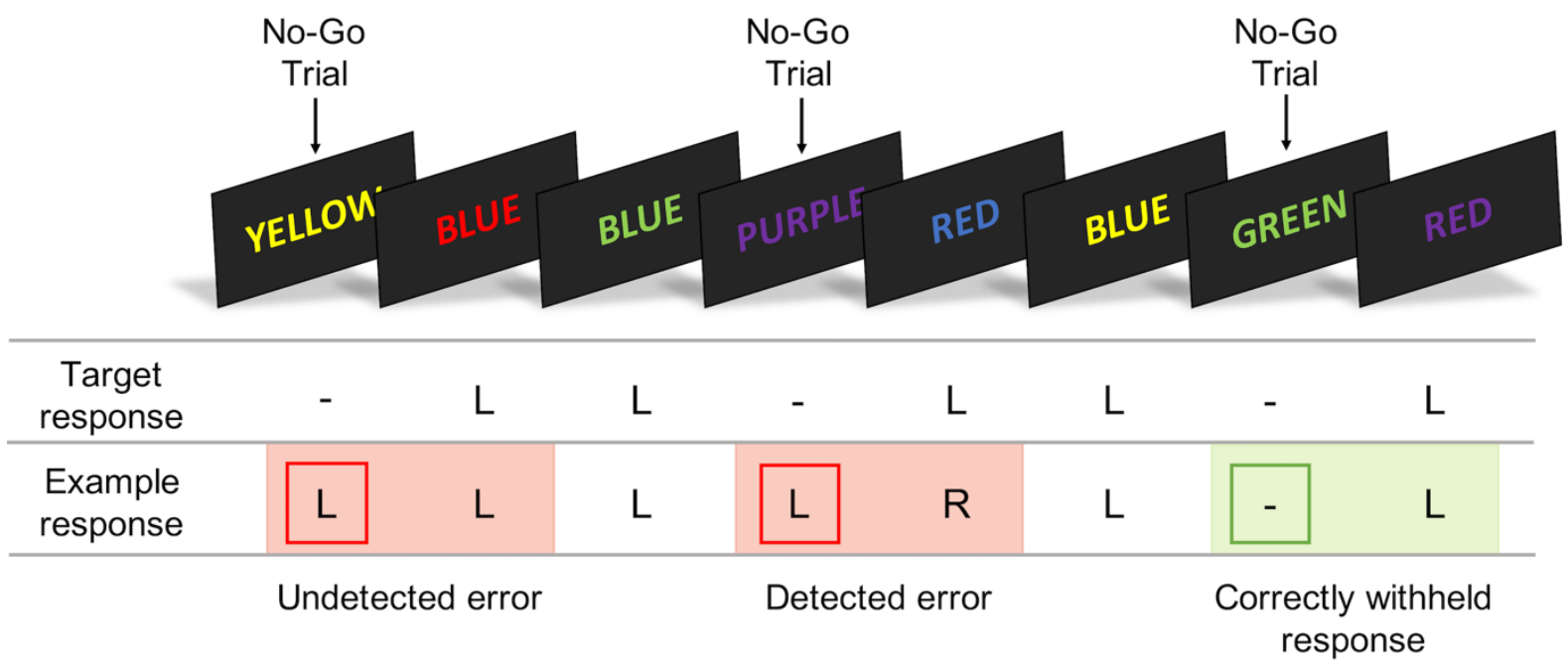

Note. The error awareness task presents a serial stream of colour words, in a congruent (No-

179 Go trial) or incongruent (Go trial) colour. Participants respond to Go trials using a left button

180 press ('L') while withholding their response to No-Go trials. To indicate error awareness, participants forgo making a standard 'Go' response and instead execute a right button press trials. All stimuli were presented for $900 \mathrm{~ms}$ followed by a $600 \mathrm{~ms}$ inter-stimulus interval.

\section{Image Acquisition}

188 Biomedical Imaging (Victoria, Australia). Images were acquired using a Siemens Skyra 3-

189 Tesla MRI scanner with a 32-channel head coil. High resolution T1-weighted structural

190 MPRAGE images $(\mathrm{TE}=2.07 \mathrm{~ms}, \mathrm{TR}=2300 \mathrm{~ms}, \mathrm{FOV}=256 \mathrm{~mm}$, flip angle $=9$ degrees,

191 thickness $=1 \mathrm{~mm}$ isotropic, sagittal slices) were acquired prior to functional imaging to

192 enable activation localisation and for spatial normalisation. Functional images were acquired

193 using a gradient-echo pulse (EPI) sequence $(\mathrm{TE}=30 \mathrm{~ms}, \mathrm{TR}=2460 \mathrm{~ms}, \mathrm{FOV}=190 \mathrm{~mm}$,

194 flip angle $=90$ degrees, 44 contiguous transversal slices of $3.0 \mathrm{~mm}$ thickness). The error 
awareness task was presented using E-Prime software (version 2.0; Psychology Software

197 positioned in the radio frequency head coil. Participants responded to each stimulus using

198 their right hand, entering their responses using two buttons on a four-button MR-compatible

199 response pad (Fiber-Optic response pads; Current Designs).

\section{$200 \quad$ Statistical Analysis}

Behavioural Analysis

Behavioural data analysis was undertaken in the programming language $\mathrm{R}$ using the stats package (R Core Team 2017), with the addition of the psych (Revelle 2020), afex

204 (Singmann et al. 2020) and emmeans (Russell et al. 2020) packages. Effect sizes were calculated using the effectsize package (Ben-Shachar et al. 2020). Assumptions were tested, and non-parametric analyses were computed under violations of normality. Greenhouse-

Geisser-adjusted degrees of freedom and $p$-values are reported under violations of sphericity. Post-hoc tests were undertaken using Tukey's method for multiple comparisons. $P$-values were otherwise adjusted using Holm procedures. Alpha was set to .05 for all analyses. The

210 number of trials available for our behavioural analyses are outlined in Table $3 \mathrm{~A}$ of the

211 Supplementary Material. The full reproducible code for the current results has been made

212 publicly available online (https://osf.io/hrba7/).

The error awareness task is not optimised to analyse response speed adjustments

214 following errors as participants are required to make an awareness button press on the first

215 post-error trial. Switching to the awareness button typically results in abnormally fast

216 reaction times on the Go trials following the error. Response speed adjustments following

217 No-Go trials were therefore determined by calculating the difference in reaction time for the

218 Go trial following the No-Go trial by three trials and the Go trial immediately preceding the 
219 No-Go trial (a subtraction of the pre-error Go reaction time from the third Go reaction time

220 after the No-Go trial).

\section{$221 \quad$ Neuroimaging Analysis}

Neuroimaging analyses were undertaken using AFNI software (Cox 1996). Data

analysis procedures followed those implemented in studies with similar experimental

paradigms (e.g., Hester et al. 2012). Behavioural data were used to categorise trial events into

the following regressors: correct inhibitions, unaware errors and aware errors. Activation

outside of the brain was removed using edge detection techniques. Following image

reconstruction, the time series data were time shifted (using Fourier interpolation) to remove

differences in slice acquisition times and then motion corrected using 3D volume registration

(least-squares alignment of three translational and three rotational parameters).

231 functions (IRFs) were computed at $2.46 \mathrm{~s}$ temporal resolution for aware errors, unaware

232 errors and correct inhibitions. To avoid confounding the baseline and event-related activity

233 estimates, rest and omission errors were included as regressors of no interest. A multiple

234 regression program (3dDeconvolve) determined the best fitting gamma variate function for

235 these IRFs. The area under the curve of the gamma variate function was expressed as a

236 percentage of the area under the baseline. The baseline in this design refers to task-related

237 Go-trial processing that remains once the variance of the other events has been removed. The

238 percentage area (event-related activation) map voxels were re-sampled at $1 \mathrm{~mm}$ resolution,

239 then spatially normalised to standard MNI space and spatially blurred with a $3 \mathrm{~mm}$ isotropic

240 root mean squared Gaussian kernel.

241 Group activation maps were obtained using a paired samples $t$-test $(3 \mathrm{dttest}++)$ against

242 the null hypothesis of no event-related activation differences between aware and unaware

243 errors. Significant voxels passed a voxel-wise statistical threshold $\left(t=6.60, p=1.0 \times 10^{-10}\right)$ 
and were required to be part of a $250 \mu 1$ cluster of significant contiguous voxels. This method

245 of combining probability and cluster thresholding sought to maximise power while

246 minimising the likelihood of false-positives. ANFI's 3dClustSim was provided with the

247 number of voxels in the group map, the spatial correlation of the voxels, and the voxel-wise

248 threshold. A series of Monte Carlo simulations (10,000 iterations) were then undertaken to

249 determine the frequency of varying sized clusters produced by chance. From this frequency

250 distribution, we selected the cluster size that occurred less than $1 \%$ of the time by chance, to

251 provide a threshold of $p=.01$, corrected. Using this method for the current sample resulted in

252 a highly liberal cluster-wise threshold $(<1 \mu \mathrm{l})$. We thus opted for a cluster-wise threshold of

$253250 \mu \mathrm{l}$ as it is far more conservative and is moreover comparable with previous studies (e.g.,

254 Hester et al. 2005). Mean activity estimates for each event were derived for clusters in the

255 whole brain map using the program 3DRoiStats. The estimates were used in assessing the

256 relationship between neural activity and measures of task performance and individual

257 differences.

\section{Linking Neural Activity to Psychopathological Traits}

Lasso (least absolute shrinkage and selection operator) regression was employed to

determine a subset of the dimensional psychopathological measures that best predict error

awareness and mean activity estimates for the supramarginal gyrus, insula and anterior

cingulate cortices. Lasso is a modified form of least squares regression that applies a

regularisation parameter $(\lambda)$ to determine the variables that best predict the outcome measure

264 (Tibshirani 1996). The regularisation parameter shrinks coefficients to zero for irrelevant covariates in order to minimise prediction error and reduce overfitting. The optimal penalty

267 provides a principled way of identifying a subset of predictors that have the strongest

268 influence on the dependent variable (Tibshirani 1996). Lasso generalised linear models were 
computed in the programming language $\mathrm{R}$ (R Core Team 2017) using the glmnet package

270 (Friedman et al. 2010). The main independent variables were subscale scores from each of

271 the aforementioned psychopathological questionnaires. Although a whole-brain approach was

272 used to explore the regions associated with awareness, a more focused subset of areas was

273 selected as dependent variables to investigate how awareness activity is related to

274 psychopathological traits. Dependent variables were therefore error awareness, and mean

275 aware activity estimates for three clusters identified in our imaging analysis (anterior

276 cingulate cortex, insula cortex and supramarginal gyrus). These clusters were selected due to

277 theoretical relevance and previous findings of sensitivity to error awareness (Harsay et al.

278 2012; Orr and Hester 2012; Klein et al. 2013b). Four separate models were computed, one for

279 each dependent variable. The analysis does not allow missing data, therefore cases with

280 missing values were omitted. Little's test indicated that data were missing completely at

281 random, $\chi^{2}(155)=173.53, p=.155$. All variables were standardised prior to analysis to

282 generate $Z$-scores. A test statistic or $p$-value for lasso regression is still under development

283 (Lockhart et al. 2014). Further, given the interest here is predictive performance and not

284 statistical inference, results are presented as standardised regression weights alone. To

285 determine the robustness of the variable selection, each Lasso model was computed on 500

286 bootstrap samples. The percentage of non-zero bootstrap samples is reported for each

287 variable alongside the coefficients.

Results

289 Behavioural Results

Performance indices are summarised in Table 1. Participants correctly withheld

$29153.59 \%$ of their responses on No-Go trials, and were aware of $86.47 \%$ of commission errors

292 (error awareness range 21.74-99.11\%). There was a non-significant weak association

293 between awareness of errors and overall inhibition performance, $r_{s}=-.10, p=.055$. A 
repeated measures ANOVA revealed that the speed of response was significantly related to

295 trial type, $F(2,798)=21.37, p<.001, \eta_{p}{ }^{2}=.05$. Post-hoc tests using the Tukey method for

296 multiple comparisons indicated that reaction times were significantly faster for aware errors

297 than for either unaware errors, $t(798)=-4.22, p<.001, d=-.23,95 \% \mathrm{CI}[-.30,-.16]$, or

298 correct Go responses, $t(798)=-6.44, p<.001, d=-.15,95 \%$ CI [-.22, -.08]. There was no

299 significant difference in reaction time between correct responses and unaware errors, $t(798)=$

300

$2.22, p=.069, d=-.08,95 \%$ CI $[-.15,-.01]$.

A repeated measures ANOVA was computed to compare reaction time adjustments

302 across No-Go responses (correct inhibitions, aware error, unaware error). The results

303 revealed an effect of No-Go response type on post-No-Go reaction time, $F(1.52,598.43)=$

$3044.85, p=.015, \eta_{p}^{2}=.01$. Post-hoc tests indicated greater slowing of responses following

unaware errors $(+19 \mathrm{~ms})$ compared to aware errors $(+5 \mathrm{~ms}), t(786)=2.91, p=.010, d=-.48$,

306

$95 \%$ CI $[-.56,-.41]$, and correct No-Go responses $(+7 \mathrm{~ms}), t(786)=2.41, p=.043, d=-.40$,

95\% CI [-.47, -.33]. There was no significant difference in post-No-Go reaction adjustments

308 between correct responses and aware errors, $t(786)=0.50, p=.869, d=.08,95 \% \mathrm{CI}[.01$, $309.15]$

310 


\section{Table 1}

313 Behavioural Performance: Inhibition Accuracy, Error Awareness and Reaction Time on the

314 Error Awareness Task

\begin{tabular}{lc}
\hline Category & Mean $(S D)$ \\
\hline Inhibition accuracy \% & $53.59(19.31)$ \\
Total errors & $66.45(29.05)$ \\
Error awareness \% & $86.47(11.74)$ \\
Reaction time (ms) & \\
$\quad$ Go trial & $517.66(80.60)$ \\
$\quad$ Aware trial & $489.14(107.28)$ \\
$\quad$ Unaware trial & $507.37(117.33)$ \\
Post-No-Go reaction time adjustment (ms) ${ }^{\mathrm{a}}$ & \\
$\quad$ Aware error & $+5.94(49.66)$ \\
$\quad$ Unaware error & $+19.22(93.21)$ \\
$\quad$ Correct inhibition & $+7.89(44.24)$ \\
\hline
\end{tabular}

315 apost-No-Go reaction time - pre-No-Go reaction time. Post-No-Go reaction time is taken

316 from the Go trial that succeeds the No-Go by three trials.

318 Neuroimaging Results

319 The event-related functional analysis revealed 17 clusters that differentiated aware

320 errors from unaware errors (Table 2). Aware errors were accompanied by greater activity in

321 the left insula cortex (Figures 2C and 2D), the supramarginal gyrus (SMG; Figure 2B), and

322 midline structures such as the left supplementary motor area (SMA), left anterior cingulate

323 cortex (ACC) and bilateral precuneus (Figure 2A). It should be noted that while the centre of

324 mass of activity in the SMA and ACC falls within the left hemisphere, the lateral extent of

325 these clusters was bilateral.

326 ACC activity was not robustly correlated with behavioural measures that are typically

327 related to error awareness. That is, the speed of error commission was not significantly 
associated with the degree of ACC activity for either aware, $r_{s}=-.09, p=.061$, or unaware between inhibition performance and ACC activity related to aware errors, $r_{s}=.01, p=.890$, nor error awareness rate, $r_{s}=.09, p=.063$. Likewise, we found no evidence for an association between post-error reaction time adjustments and ACC activity for aware errors, $r_{s}=-.06, p=.233$, or unaware errors, $r_{s}=-.08, p=.096$. The difference between BOLD activity in the ACC associated with aware errors and that associated with unaware errors was also not found to be significantly related to the speed of aware errors, $r_{s}=-.02, p=1.00$, and unaware errors, $r_{s}=.01, p=1.00$, or post-aware error adjustments in reaction time following aware errors, $r_{s}=-.03, p=1.00$, and unaware errors, $r_{s}=.09, p=.284$. Inhibition performance was found to correlate positively with BOLD activity associated with 340 aware errors in the insula, $r_{s}=.12, p=.030$, and right SMG, $r_{s}=.25, p<.001$. Only the left

341 middle frontal gyrus, $r_{s}=.17, p=.010$, and the SMA, $r_{s}=.15, p=.030$, were found to

342 correlate significantly with aware error reaction time. Post-aware reaction time adjustments

343 were associated with activity in the SMA, $r_{s}=.15, p=.030$, right SMG, $r_{s}=.19, p<.001$,

344 right superior temporal gyrus, $r_{s}=.14, p=.040$, and left and right middle frontal gyri, $r_{s}=$ $345.17, p=.010$, and $r_{s}=.21, p<.001$, respectively, such that greater activity in these regions 346 correlated with slower reaction time on the post-error trial. For unaware errors, neither the

347 speed of the erroneous response nor post-error reaction time adjustments were found to

348 significantly correlate with BOLD activity in any of the 17 clusters. 
Figure 2
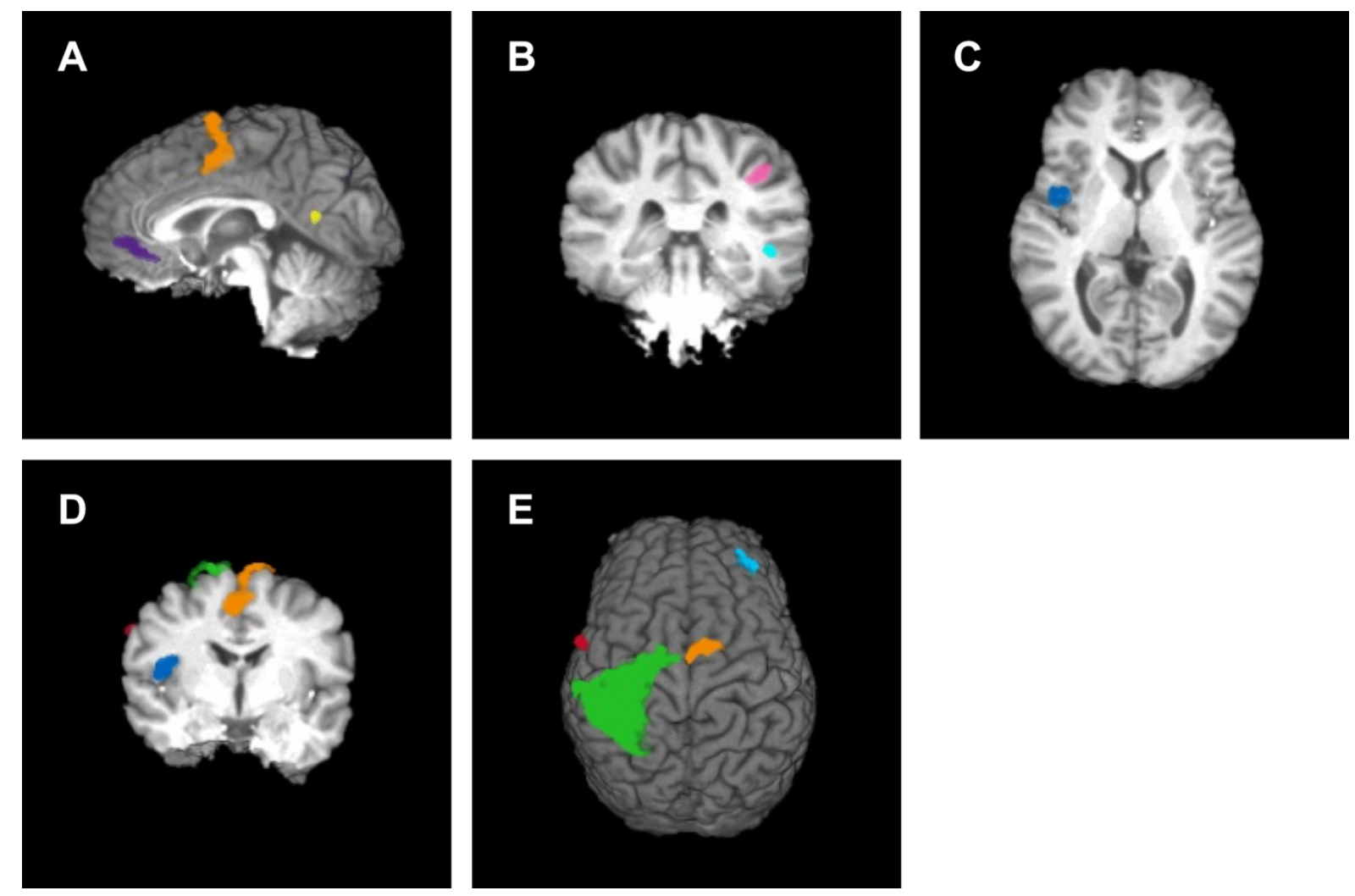

353 Note. Clusters associated with greater BOLD signal for aware errors than unaware errors. (A)

354 Sagittal slice at $x=0$. Purple cluster centred on left anterior cingulate cortex, orange cluster

355 centred on left supplementary motor area (SMA), yellow cluster centred on left precuneus;

356 (B) Blue cluster centred on right superior temporal gyrus, pink cluster centred on right

357 supramarginal gyrus; (C) Axial slice at $z=10$. Blue cluster centred on left insula; (D)

358 Coronal slice at $y=5$. Blue cluster centred on left insula, red cluster centred on left precentral

359 gyrus, orange cluster centred on left SMA, green cluster centred on left postcentral gyrus; (E)

360 Green cluster centred on right postcentral gyrus, orange cluster centred on left SMA, red

361 cluster centred on left precentral gyrus, blue cluster centred on right middle frontal gyrus. 
Table 2

364 Regions that Showed Significantly Greater BOLD Signal for Aware Errors than Unaware

365 Errors

\begin{tabular}{|c|c|c|c|c|c|}
\hline \multirow[t]{2}{*}{ Structure } & \multirow[t]{2}{*}{ Vol. $\mu 1$} & \multirow[t]{2}{*}{ HS } & \multicolumn{3}{|c|}{ Centre of mass (MNI Coordinates) } \\
\hline & & & $x$ & $y$ & $z$ \\
\hline $\begin{array}{l}\text { Postcentral } \\
\text { gyrus }\end{array}$ & 30420 & $\mathrm{~L}$ & -38 & -32 & 54 \\
\hline Cerebellum & 7778 & $\mathrm{R}$ & 23 & -50 & -27 \\
\hline Cerebellum & 4055 & $\mathrm{~L}$ & -30 & -52 & -31 \\
\hline $\mathrm{SMA}^{\mathrm{a}}$ & 2849 & $\mathrm{~L}$ & 1 & -6 & 58 \\
\hline Cerebellum & 2401 & $\mathrm{R}$ & 21 & -49 & -57 \\
\hline $\mathrm{ACC}^{\mathrm{a}}$ & 1784 & $\mathrm{~L}$ & -2 & 41 & -3 \\
\hline Insula & 1521 & $\mathrm{~L}$ & -40 & -2 & 13 \\
\hline SMG & 1368 & $\mathrm{R}$ & 39 & -37 & 43 \\
\hline Cerebellum & 1085 & $\mathrm{~L}$ & -34 & -44 & -59 \\
\hline $\begin{array}{l}\text { Middle frontal } \\
\text { gyrus }\end{array}$ & 703 & $\mathrm{~L}$ & -32 & 37 & 35 \\
\hline $\begin{array}{l}\text { Middle frontal } \\
\text { gyrus }\end{array}$ & 687 & $\mathrm{R}$ & 32 & 44 & 38 \\
\hline SMG & 585 & $\mathrm{R}$ & 56 & -43 & 26 \\
\hline Precuneus & 554 & $\mathrm{R}$ & 7 & -73 & 42 \\
\hline Precuneus & 541 & $\mathrm{~L}$ & -5 & -57 & 13 \\
\hline $\begin{array}{l}\text { Superior } \\
\text { temporal gyrus }\end{array}$ & 394 & $\mathrm{R}$ & 46 & -29 & -3 \\
\hline Precentral gyrus & 375 & $\mathrm{~L}$ & -57 & 2 & 33 \\
\hline Precuneus & 252 & $\mathrm{~L}$ & -11 & -70 & 47 \\
\hline
\end{tabular}

366 Note. Positive values for $x, y$ and $z$ coordinates denote locations that are right, posterior and

367 superior relative to the anterior commissure, respectively. SMA, supplementary motor area;

368 ACC, anterior cingulate cortex; SMG, supramarginal gyrus.

$369{ }^{\text {a }}$ While the centre of mass of activity in the SMA and ACC falls within the left hemisphere,

370 the lateral extent of these clusters was bilateral. 


\section{Lasso Regression}

373 the Supplementary Material for descriptive statistics). Error awareness was found to be

374 predicted by impulsivity, namely motor and planning scores from the BIS-11, and

375 behavioural inhibition score from the BIS/BAS (Carver and White 1994). The most important

376 predictors of insula activity were attention to detail and imagination scores from the AQ

377 (Baron-Cohen et al. 2001), and HADS depression score (Zigmond and Snaith 1983). No

378 variable was found to predict ACC activity, while all variables except attention switching

379 from the AQ, and attention, self-concept and DSM attention from the CAARS (Conners

380 1998) were found to predict SMG activity. 
382 Table 3

383 Lasso Regression Coefficients

\begin{tabular}{|c|c|c|c|c|c|c|c|c|c|c|}
\hline & \multicolumn{10}{|c|}{ Outcome } \\
\hline & \multicolumn{2}{|c|}{$\begin{array}{c}\text { Error } \\
\text { awareness }\end{array}$} & \multicolumn{2}{|c|}{ L-insula } & \multicolumn{2}{|c|}{$\mathrm{ACC}$} & \multicolumn{2}{|c|}{ R-SMG } & \multicolumn{2}{|c|}{$\begin{array}{l}\text { R-Middle } \\
\text { frontal gyrus }\end{array}$} \\
\hline Predictor & $\begin{array}{c}\text { LASSO } \\
\text { coeff. }\end{array}$ & $\begin{array}{l}\text { non- } \\
\text { zero } \\
(\%)\end{array}$ & $\begin{array}{c}\text { LASSO } \\
\text { coeff. }\end{array}$ & $\begin{array}{l}\text { non- } \\
\text { zero } \\
(\%)\end{array}$ & $\begin{array}{c}\text { LASSO } \\
\text { coeff. }\end{array}$ & $\begin{array}{l}\text { non- } \\
\text { zero } \\
(\%)\end{array}$ & $\begin{array}{l}\text { LASSO } \\
\text { coeff. }\end{array}$ & $\begin{array}{l}\text { non- } \\
\text { zero } \\
(\%)\end{array}$ & $\begin{array}{c}\text { LASSO } \\
\text { coeff. }\end{array}$ & $\begin{array}{l}\text { non- } \\
\text { zero } \\
(\%)\end{array}$ \\
\hline $\begin{array}{l}\text { BIS-11 - } \\
\text { attentional }\end{array}$ & . & 56 & . & 56 & . & 26 & -0.05 & 58 & . & 33 \\
\hline BIS-11-motor & -0.07 & 86 & . & 46 & . & 30 & 0.11 & 78 & 0.05 & 74 \\
\hline $\begin{array}{l}\text { BIS-11 - non- } \\
\text { planning }\end{array}$ & -0.03 & 90 & . & 38 & . & 48 & -0.01 & 52 & . & 34 \\
\hline $\begin{array}{l}\mathrm{AQ} \text { - social } \\
\text { skill }\end{array}$ & . & 48 & . & 40 & . & 56 & -0.15 & 82 & . & 46 \\
\hline $\begin{array}{l}\mathrm{AQ} \text { - attention } \\
\text { switching }\end{array}$ & . & 68 & . & 50 & . & 22 & . & 54 & . & 37 \\
\hline $\begin{array}{l}\mathrm{AQ}-\text { attention } \\
\text { to detail }\end{array}$ & . & 64 & .0003 & 60 & . & 44 & 0.04 & 64 & . & 47 \\
\hline $\begin{array}{l}\mathrm{AQ}- \\
\text { communication }\end{array}$ & . & 58 & . & 56 & . & 20 & 0.16 & 92 & 0.05 & 71 \\
\hline $\begin{array}{l}\mathrm{AQ}- \\
\text { imagination }\end{array}$ & . & 54 & 0.02 & 76 & . & 34 & 0.03 & 64 & 0.13 & 92 \\
\hline $\begin{array}{l}\text { BIS/BAS - } \\
\text { BAS drive }\end{array}$ & . & 68 & . & 56 & . & 30 & 0.04 & 66 & 0.05 & 72 \\
\hline $\begin{array}{l}\text { BIS/BAS - } \\
\text { BAS fun }\end{array}$ & . & 50 & . & 42 & . & 24 & -0.12 & 74 & . & 25 \\
\hline $\begin{array}{l}\text { BIS/BAS - } \\
\text { BAS reward }\end{array}$ & . & 62 & . & 42 & . & 34 & 0.01 & 54 & . & 41 \\
\hline $\begin{array}{l}\text { BIS/BIS - BIS } \\
\text { score }\end{array}$ & 0.04 & 90 & . & 68 & . & 30 & 0.03 & 60 & 0.01 & 53 \\
\hline
\end{tabular}




\begin{tabular}{|l|c|c|c|c|c|c|c|c|c|c|}
\hline $\begin{array}{l}\text { HADS - } \\
\text { anxiety }\end{array}$ & $\cdot$ & 52 & $\cdot$ & 50 & $\cdot$ & 30 & 0.02 & 64 & $\cdot$ & 49 \\
\hline $\begin{array}{l}\text { HADS - } \\
\text { depression }\end{array}$ & $\cdot$ & 52 & 0.10 & 90 & $\cdot$ & 34 & 0.19 & 98 & $\cdot$ & 79 \\
\hline $\begin{array}{l}\text { CAARS - } \\
\text { attention }\end{array}$ & $\cdot$ & 42 & $\cdot$ & 38 & $\cdot$ & 22 & $\cdot$ & 38 & $\cdot$ & 43 \\
\hline $\begin{array}{l}\text { CAARS - } \\
\text { hyperactivity }\end{array}$ & $\cdot$ & 46 & $\cdot$ & 52 & $\cdot$ & 24 & 0.11 & 54 & $\cdot$ & 25 \\
\hline $\begin{array}{l}\text { CAARS - } \\
\text { impulsivity }\end{array}$ & $\cdot$ & 48 & $\cdot$ & 48 & $\cdot$ & 22 & -0.04 & 60 & $\cdot$ & 37 \\
\hline $\begin{array}{l}\text { CAARS - self- } \\
\text { concept }\end{array}$ & $\cdot$ & 46 & $\cdot$ & 42 & $\cdot$ & 54 & $\cdot$ & 36 & $\cdot$ & 32 \\
\hline $\begin{array}{l}\text { CAARS - DSM } \\
\text { attention }\end{array}$ & $\cdot$ & 34 & $\cdot$ & 50 & $\cdot$ & 20 & $\cdot$ & 42 & -0.05 & 49 \\
\hline $\begin{array}{l}\text { CAARS - DSM } \\
\text { hyperactivity }\end{array}$ & $\cdot$ & 38 & $\cdot$ & 40 & $\cdot$ & 22 & -0.14 & 60 & $\cdot$ & 23 \\
\hline $\begin{array}{l}\text { CAARS - DSM } \\
\text { ADHD }\end{array}$ & $\cdot$ & 6 & $\cdot$ & 4 & $\cdot$ & 6 & -0.01 & 20 & $\cdot$ & 24 \\
\hline $\begin{array}{l}\text { CAARS - } \\
\text { index }\end{array}$ & $\cdot$ & 64 & $\cdot$ & 18 & $\cdot$ & 12 & -0.02 & 40 & $\cdot$ & 24 \\
\hline
\end{tabular}

384 Note. Results are presented as standardised regression coefficients. To determine the

385 robustness of the variable selection, each Lasso model was computed on 500 bootstrap

386 samples. The percentage of non-zero bootstrap samples is reported for each variable

387 alongside the coefficients. BIS, Barratt Impulsiveness Scale, Version 11; AQ, Autism

388 Spectrum Quotient; BIS/BAS, Behavioural Inhibition/Avoidance Scale; HADS, Hospital

389 Anxiety and Depression Scale; CAARS, Conners' Adult ADHD Rating Scale; ACC, anterior

390 cingulate cortex; SMG, supramarginal gyrus. 


\section{Discussion}

The current study aimed to establish the robustness of previous findings on the neural

394 correlates of error awareness. Here, we have discerned greater aware-related activity in a

395 network of regions including the insula cortex, anterior cingulate cortex (ACC),

396 supplementary motor area (SMA), and supramarginal gyrus (SMG). Further, individual

397 differences in error-related neural activity were found to be related to indices of task

398 performance in a select few regions including the insula, SMA and SMG. Moreover, we

399 found that certain measures of psychopathology - namely impulsiveness and depression -

400 explained variance in aware-related activity in a subset of these regions.

401

Although the ACC has been implicated in several studies on performance monitoring,

differentiation of activity in this region with error awareness has been largely unreported (but

see Hester et al., 2012). Our study has shown greater ACC activation - across the dACC and

rACC - for aware errors than unaware errors, suggesting a sensitivity of the ACC to

awareness. This supports claims that insufficient statistical power may underlie the

discrepancy in previous findings (Wessel, 2012). Although the precise role of the ACC in

407 error processing is unknown, there is a general consensus that the ACC - particularly the

408 dACC - monitors ongoing behaviour and navigates the selection and evaluation of goal-

409 directed behaviours (Holroyd and Yeung 2012). In particular, it is purported to respond to

410 outcomes that are worse than expected and may signal the need for an adjustment in strategy

411 to reach the desired goal (Holroyd and Coles 2002; Bryden et al. 2011). Our finding of

412 greater rACC activation is not typically reported in error awareness studies, however the

413 rACC has been proposed to be a neuronal generator of the $\mathrm{Pe}-$ an event-related potential

414 associated with error awareness (Herrmann et al. 2004; Van Boxtel et al. 2005). While the

415 rACC may be differentially involved in post-error processing, as evidenced by the Pe, further

416 work is ultimately needed to discern precisely how the rACC contributes to awareness. Taken 
417 together, it is plausible that the ACC presents a threshold-like relationship to awareness and

418 post-error processes, whereby a certain level of activity is sufficient to elicit error detection

419 and post-error adaptation, but the overall level of activity is not tightly coupled to these

420 processes (Orr and Hester 2012). This is moreover consistent with the absence of a

421 relationship between individual differences in aware-related ACC activity and behavioural

422 adjustments in our study. Thus, ACC activity may covary with error commission and

423 contribute to error awareness such that it is facilitating goal attainment, however may not be

424 solely responsible for eliciting awareness and post-error alterations.

425 Contrastingly, the insula cortex appears to be consistently modulated by error

426 awareness. The insula has been proposed to be engaged in a number of processes, however its

427 role in interoceptive awareness has taken prominence in recent decades. In particular, the

428 insula integrates autonomic information with salient events such as errors (Klein et al.

429 2013b). Activity of the insula during aware errors may therefore be explained by

430 interoceptive awareness of greater autonomic responses to aware errors (Craig 2009).

431 Interestingly, concurrent insula and ACC activity during performance monitoring is a robust

432 finding (Craig 2009; Ham et al. 2013). Although these structures are distinct, they have been

433 purported to form a salience network which has been associated with interoceptive autonomic

434 domains and the control of goal-directed behaviours (Dosenbach et al. 2007). Indeed,

435 previous studies have found the ACC to be associated with autonomic engagement during

436 aware error processing (Harsay et al. 2018). The relationship between the ACC and insula

437 may explain how the ACC potentially mediates error awareness. Specifically, insula activity

438 may represent awareness while ACC activity represents the control of directed effort. That is,

439 error-related activity in the ACC may feedforward into the insula which may be more directly

440 responsible for error awareness. 
442 in the right SMG. The SMG is purported to be connected to the ACC and middle frontal

443 cortex via the dorsal branch of the superior longitudinal fasciculus (SLF1; Ramos-Fresnedo

444 et al. 2019). Recent research has demonstrated that individual differences in the SLF1

445 underpin an individual's evidence accumulation capacity (Brosnan et al. 2020). This is

446 pertinent given that current views on error awareness operate in line with an evidence

447 accumulation account (Ullsperger et al. 2010). The emergence of error awareness is said to

448 coincide with the accumulation of evidence above a response criterion threshold (Murphy et

449 al. 2012). Given the SMG, ACC and middle frontal cortex were found to contribute to error

450 awareness in the current study, it is plausible that connectivity between these regions might

451 be a critical determinant of an individual's error awareness.

It is also worth considering that the inferior parietal lobe - which in part comprises

453 the SMG - has been proposed to form a network with the ACC and insula and together are

454 associated with the salience of an event (Harsay et al., 2012). The parietal lobe, in particular,

455 is suggested to act on salient events and likely works to direct and maintain the location of

456 attention (Corbetta and Shulman 2002). Errors are arguably salient as they are infrequent and

457 useful in that they re-direct a participant's attention to current task goals. Indeed, consistent

458 with this orienting account, we found elevated SMG activity to be correlated with slower

459 reaction times following aware errors. This finding aligns with previous work which has

460 found that correct trials following an error show heightened activation of the inferior parietal

461 lobe, coinciding with increased post-error slowing (Marco-Pallarés et al. 2008).

Although error awareness rate did not appear to be associated with inhibition

463 performance, we found a relationship between inhibition and aware-related activity in the

464 insula and SMG. This is interesting given that error-related activity in the insula and inferior

465 parietal lobe have previously been found to predict successful inhibition on the following No- 
466 Go trial (Hester et al. 2009b), suggesting a shared neural system between error awareness and

467 successful response inhibition. Previously, we speculated that inclusion of two inhibition

468 contingencies might disrupt the relationship between error awareness and future performance,

469 reflecting the role of the ACC as a reinforcement learning signal (Orr and Hester 2012). We

470 therefore opted to include only one inhibition rule in the current design. Despite this task

471 change, we found no relationship between error awareness and inhibition performance. It is

472 thus plausible that error awareness facilitates performance only under context-specific

473 conditions - where there is a more direct contingency between an error and future

474 performance. Since there was no direct contingency here, with performance not influencing

475 the sequence of trials that followed, it is likely any increases in conservatism of responding

476 are loosely, if at all, reflective of sustained changes in performance strategy.

477 While we examined post-error reaction time adjustments, it is worth considering that

478 the error awareness task is not optimised for this analysis. Specifically, participants are

479 required to make an awareness button press on the first post-error trial. To minimise this

480 confound, we excluded the first two post-error trials from our post-error slowing analysis,

481 however we still found greater slowing following unaware errors. While studies on post-error

482 slowing and error awareness have generated mixed evidence (van Gaal et al. 2009; Hewig et

483 al. 2011; Endrass et al. 2012; Hoonakker et al. 2016), the finding of greater slowing

484 following unaware errors appears to be exclusive to studies employing the error awareness

485 task. Given that post-error reaction time did not return to baseline by the third post-error trial,

486 it seems plausible that unaware errors are accompanied by the continued anticipation of an

487 impending No-Go trial, resulting in slowed responses. Our finding of greater slowing

488 following unaware errors is therefore likely to be a task-specific phenomenon rather than a

489 reflection of deliberate post-error behavioural adjustments. To reconcile these findings, we 
require a task that obviates the need for an error awareness button press on the post-error trial and offers more events (i.e., aware and unaware errors) per individual. awareness and related neural activity in three selected clusters (ACC, insula and SMG), we ran a series of Lasso regressions. The most robust positive predictors of error awareness were impulsivity-related measures, specifically motor and non-planning impulsiveness. This is consistent with the finding that disorders marked by deficits in impulsiveness, such as ADHD and substance use disorder, have been shown to have impaired error awareness (O'Connell et al. 2009; Charles et al. 2017). Error awareness was also found to be positively predicted by behavioural inhibition system score which reflects the motivation to avoid adverse outcomes and is purported to be predictive of affective and behavioural responses after incentives and threats (Johnson et al. 2003). Moreover, the relationship between aware-related insula and

502 SMG activity was found to be most notably positively predicted by depressive symptoms.

503 Although some studies have found a heightened error positivity - an event-related potential

504 which is suggested to index error awareness - to be related to depressive symptoms (Mies et

505 al. 2011; Mueller et al. 2015), others have found no such relationship (Compton et al. 2008).

506 It has been reported, however, that depressed individuals display greater activity in the insula

507 in response to negative stimuli than healthy controls (Hamilton et al. 2012). The heightened

508 sensitivity to failure and negative information which is proposed to underlie clinical levels of

509 depression may in part explain why aware-related activity in these regions is related to

510 depressive traits in a non-clinical sample.

511 Our event-related analysis of a large sample revealed a network of regions including

512 the insula cortex, SMG, and midline structures such as the ACC and SMA that show greater

513 BOLD signal change for aware errors compared to unaware errors. The most parsimonious 
514 account of error awareness is that it is likely the result of the accumulative efforts of these

515 systems which may not all individually drive awareness.

516

517 Open practices statement. Data are available upon reasonable request and all scripts

518 required for the current results have been made publicly available online at the Open Science

519 Framework (https://osf.io/hrba7/).

520 Conflict of interest. The authors declare no conflicts of interest.

521 Acknowledgements. This work was supported by a Project Grant from the National Health

522 and Medical Research Council (NHMRC) of Australia to M.A.B and R.H (\#1045354).

523 M.A.B is supported by a Senior Research Fellowship (Level B) from the NHMRC (\#

524 1154378). This work was also supported by a Marie Skłodowska-Curie Fellowship from the

525 European Commission (AGEING PLASTICITY; grant number 844246) to M.B, and

526 supported by the NIHR Oxford Health Biomedical Research Centre. The Wellcome Centre

527 for Integrative Neuroimaging is supported by core funding from the Wellcome Trust

$528(203139 / Z / 16 / Z)$. For the purpose of open access, the author has applied a CC BY public

529 copyright licence to any Author Accepted Manuscript version arising from this submission.

530 We would like to thank Mr Cameron Patrick for his assistance with the Lasso regression. 
533 Baron-Cohen S, Wheelwright S, Skinner R, Martin J, Clubley E. 2001. The Autism-Spectrum

534 Quotient (AQ): Evidence from Asperger Syndrome/High-Functioning Autism, Males and

535 Females, Scientists and Mathematicians. J Autism Dev Disord. 31:5-17.

536 Barratt ES, Patton JH. 1983. Impulsivity: Cognitive, behavioral, and psychophysiological

537 correlates. In: Zuckerman M, editor. Biological bases of sensation seeking, impulsivity and

538 anxiety Hillsdale, NJ: Erlbaum p 77-116.

539 Ben-Shachar M, Lüdecke D, Makowski D. 2020. effectsize: Estimation of effect size indices

540 and standardized parameters. J Open Source Softw. 5:2815.

541 Bossier H, Roels SP, Seurinck R, Banaschewski T, Barker GJ, Bokde ALW, Quinlan EB,

542 Desrivières S, Flor H, Grigis A, Garavan H, Gowland P, Heinz A, Ittermann B, Martinot J-L,

543 Artiges E, Nees F, Orfanos DP, Poustka L, Fröhner Dipl-Psych JH, Smolka MN, Walter H,

544 Whelan R, Schumann G, Moerkerke B. 2020. The empirical replicability of task-based fMRI

545 as a function of sample size. NeuroImage. 212:116601.

546 Brosnan MB, Sabaroedin K, Silk T, Genc S, Newman DP, Loughnane GM, Fornito A,

547 O'Connell RG, Bellgrove MA. 2020. Evidence accumulation during perceptual decisions in

548 humans varies as a function of dorsal frontoparietal organization. Nat Hum Behav. 4:844-

549855.

550 Bryden DW, Johnson EE, Tobia SC, Kashtelyan V, Roesch MR. 2011. Attention for learning 551 signals in anterior cingulate cortex. J Neurosci. 31:18266.

552 Button KS, Ioannidis JPA, Mokrysz C, Nosek BA, Flint J, Robinson ESJ, Munafò MR. 2013.

553 Power failure: why small sample size undermines the reliability of neuroscience. Nat Rev

554 Neurosci. 14:365-376. 
555 Carver CS, White TL. 1994. Behavioral inhibition, behavioral activation, and affective

556 responses to impending reward and punishment: the BIS/BAS scales. J Pers Soc Psychol.

$557 \quad 67: 319-333$.

558 Charles L, Gaillard R, Amado I, Krebs M-O, Bendjemaa N, Dehaene S. 2017. Conscious and

559 unconscious performance monitoring: Evidence from patients with schizophrenia.

560 NeuroImage. 144:153-163.

561 Compton RJ, Lin M, Vargas G, Carp J, Fineman SL, Quandt LC. 2008. Error detection and

562 posterror behavior in depressed undergraduates. Emotion. 8:58-67.

563 Conners CK. 1998. Rating scales in attention-deficit/hyperactivity disorder: Use in

564 assessment and treatment monitoring. J Clin Psychiatry. 59:24-30.

565 Corbetta M, Shulman GL. 2002. Control of goal-directed and stimulus-driven attention in the

566 brain. Nat Rev Neurosci. 3:201-215.

567 Cox RW. 1996. AFNI: Software for analysis and visualization of functional magnetic

568 resonance neuroimages. Comput Biomed Res. 29:162-173.

569 Craig AD. 2009. How do you feel — now? The anterior insula and human awareness. Nat

570 Rev Neurosci. 10:59-70.

571 Cremers HR, Wager TD, Yarkoni T. 2017. The relation between statistical power and

572 inference in fMRI. PloS One. 12:e0184923.

573 Debener S, Ullsperger M, Siegel M, Fiehler K, von Cramon DY, Engel AK. 2005. Trial-by-

574 trial coupling of concurrent electroencephalogram and functional magnetic resonance

575 imaging identifies the dynamics of performance monitoring. J Neurosci. 25:11730-11737. 
576 Dhar M, Wiersema JR, Pourtois G. 2011. Cascade of neural events leading from error

577 commission to subsequent awareness revealed using EEG source imaging. PLoS One. 6:1-12.

578 Dosenbach NUF, Fair DA, Miezin FM, Cohen AL, Wenger KK, Dosenbach RAT, Fox MD,

579 Snyder AZ, Vincent JL, Raichle ME, Schlaggar BL, Petersen SE. 2007. Distinct brain

580 networks for adaptive and stable task control in humans. Proc Natl Acad Sci USA.

$581 \quad 104: 11073-11078$.

582 Endrass T, Klawohn J, Preuss J, Kathmann N. 2012. Temporospatial dissociation of Pe

583 subcomponents for perceived and unperceived errors. Front Hum Neurosci. 6:1-10.

584 Endrass T, Reuter B, Kathmann N. 2007. ERP correlates of conscious error recognition:

585 aware and unaware errors in an antisaccade task. Eur J Neurosci. 26:1714-1720.

586 Falkenstein M, Hohnsbein J, Hoormann J, Blanke L. 1991. Effects of crossmodal divided

587 attention on late ERP components. II. Error processing in choice reaction tasks.

588 Electroenceph Clin Neurophysiol. 78:447-455.

589 Friedman J, Hastie T, Tibshirani R. 2010. Regularization paths for generalized linear models

590 via coordinate descent. J Stat Softw. 33:1-22.

591 Gehring WJ, Goss B, Coles MGH, Meyer DE, Donchin E. 1993. A neural system for error

592 detection and compensation. Psychol Sci. 4:385-390.

593 Ham T, Leff A, de Boissezon X, Joffe A, Sharp DJ. 2013. Cognitive control and the salience

594 network: An investigation of error processing and effective connectivity. Journal of

595 Neuroscience. 33:7091-7098. 
596 Hamilton JP, Etkin A, Furman DJ, Lemus MG, Johnson RF, Gotlib IH. 2012. Functional

597 neuroimaging of major depressive disorder: A meta-analysis and new integration of baseline

598 activation and neural response data. Am J Psychiatry. 169:693-703.

599 Harsay HA, Cohen MX, Spaan M, Weeda WD, Nieuwenhuis S, Ridderinkhof KR. 2018.

600 Error blindness and motivational significance: Shifts in networks centering on anterior insula

601 co-vary with error awareness and pupil dilation. Behav Brain Res. 355:24-35.

602 Harsay HA, Spaan M, Wijnen JG, Ridderinkhof KR. 2012. Error awareness and salience

603 processing in the oddball task: Shared neural mechanisms. Front Hum Neurosci. 6:1-20.

604 Herrmann MJ, Römmler J, Ehlis A-C, Heidrich A, Fallgatter AJ. 2004. Source localization

605 (LORETA) of the error-related-negativity (ERN/Ne) and positivity (Pe). Brain Res Cogn.

$606 \quad 20: 294-299$.

607 Hester R, Foxe JJ, Molholm S, Shpaner M, Garavan H. 2005. Neural mechanisms involved in

608 error processing: A comparison of errors made with and without awareness. NeuroImage.

$609 \quad 27: 602-608$.

610 Hester R, Madeley J, Murphy K, Mattingley JB. 2009b. Learning from errors: Error-related

611 neural activity predicts improvements in future inhibitory control performance. J Neurosci.

$612 \quad 29: 7158-7165$.

613 Hester R, Nandam LS, O’Connell RG, Wagner J, Strudwick M, Nathan PJ, Mattingley JB,

614 Bellgrove MA. 2012. Neurochemical enhancement of conscious error awareness. J Neurosci.

$615 \quad 32: 2619-2627$.

616 Hester R, Nestor L, Garavan H. 2009a. Impaired error awareness and anterior cingulate

617 cortex hypoactivity in chronic cannabis users. Neuropsychopharmacology. 34:2450-2458. 
618 Hewig J, Coles MGH, Trippe RH, Hecht H, Miltner WHR. 2011. Dissociation of Pe and

619 ERN/Ne in the conscious recognition of an error. Psychophysiology. 48:1390-1396.

620 Hoffmann S, Beste C. 2015. A perspective on neural and cognitive mechanisms of error

621 commission. Front Behav Neurosci. 9:1-16.

622 Holroyd CB, Coles MG. 2002. The neural basis of human error processing: reinforcement

623 learning, dopamine, and the error-related negativity. Psychol Rev. 109:679-709.

624 Holroyd CB, Yeung N. 2012. Motivation of extended behaviors by anterior cingulate cortex.

625 Trends Cogn Sci. 16:122-128.

626 Hoonakker M, Doignon-Camus N, Bonnefond A. 2016. Performance monitoring mechanisms

627 activated before and after a response: A comparison of aware and unaware errors. Biological

628 Psychology. 120:53-60.

629 Johnson SL, Turner RJ, Iwata N. 2003. BIS/BAS levels and psychiatric disorder: An

630 epidemiological study. J Psychopathol Behav Assess. 25:25-36.

631 Klein TA, Endrass T, Kathmann N, Neumann J, von Cramon DY, Ullsperger M. 2007.

632 Neural correlates of error awareness. NeuroImage. 34:1774-1781.

633 Klein TA, Ullsperger M, Danielmeier C. 2013b. Error awareness and the insula: Links to

634 neurological and psychiatric diseases. Front Hum Neurosci. 7:14.

635 Lockhart R, Taylor J, Tibshirani RJ, Tibshirani R. 2014. A significance test for the LASSO.

636 Ann Stat. 42:413-468. 
637 Maier M, Steinhauser M, Hübner R. 2008. Is the error-related negativity amplitude related to

638 error detectability? Evidence from effects of different error types. J Cogn Neurosci. 20:2263-

6392273.

640 Marco-Pallarés J, Camara E, Münte TF, Rodríguez-Fornells A. 2008. Neural mechanisms

641 underlying adaptive actions after slips. J Cogn Neurosci. 20:1595-1610.

642 Masina F, Tarantino V, Vallesi A, Mapelli D. 2019. Repetitive TMS over the left dorsolateral

643 prefrontal cortex modulates the error positivity: An ERP study. Neuropsychologia.

$644 \quad 133: 107153$.

645 Mies GW, van der Veen FM, Tulen JHM, Birkenhäger TK, Hengeveld MW, van der Molen

646 MW. 2011. Drug-free patients with major depression show an increased electrophysiological

647 response to valid and invalid feedback. Psychol Med. 41:2515-2525.

648 Mueller EM, Pechtel P, Cohen AL, Douglas SR, Pizzagalli DA. 2015. Potentiated processing

649 of negative feedback in depression is attenuated by adhedonia. Depress Anxiety. 32:296-305.

650 Murphy P, Robertson I, Allen D, Hester R, O'Connell R. 2012. An electrophysiological

651 signal that precisely tracks the emergence of error awareness. Front Hum Neurosci. 6:65.

652 Nieuwenhuis S, Ridderinkhof KR, Blom J, Band GPH, Kok A. 2001. Error-related brain

653 potentials are differentially related to awareness of response errors: Evidence from an

654 antisaccade task. Psychophysiology. 38:752-760.

655 O'Connell RG, Dockree PM, Bellgrove MA, Kelly SP, Hester R, Garavan H, Robertson IH,

656 Foxe JJ. 2007. The role of cingulate cortex in the detection of errors with and without

657 awareness: a high-density electrical mapping study. Eur J Neurosci. 25:2571-2579. 
658 O’Connell RG, Bellgrove MA, Dockree PM, Lau A, Hester R, Garavan H, Fitzgerald M,

659 Foxe JJ, Robertson IH. 2009. The neural correlates of deficient error awareness in attention-

660 deficit hyperactivity disorder (ADHD). Neuropsychologia. 47:1149-1159.

661 Orr C, Hester R. 2012. Error-related anterior cingulate cortex activity and the prediction of 662 conscious error awareness. Front Hum Neurosci. 6.

663 Poldrack RA, Baker CI, Durnez J, Gorgolewski KJ, Matthews PM, Munafò MR, Nichols TE,

664 Poline J-B, Vul E, Yarkoni T. 2017. Scanning the horizon: Towards transparent and

665 reproducible neuroimaging research. Nat Rev Neurosci. 18:115-126.

666 R Core Team. 2017. R: A language and environment for statistical computing. R Foundation

667 for Statistical Computing.

668 Ramos-Fresnedo A, Segura-Duran I, Chaichana KL, Pillai JJ. 2019. Chapter 2 -

669 Supratentorial White Matter Tracts. In: Chaichana K, Quiñones-Hinojosa A, editors.

670 Comprehensive Overview of Modern Surgical Approaches to Intrinsic Brain Tumors

671 Academic Press p 23-35.

672 Revelle W. 2020. psych: Procedures for Personality and Psychological Research. R package

673 version 2.0.12.

674 Russell L, Henrik S, Love J, Buerkner P, Herve M. 2020. Package ‘emmeans’: Estimated

675 marginal means, aka least-squares means. R package version 1.4.8.

676 Scheffers MK, Coles MG. 2000. Performance monitoring in a confusing world: Error-related

677 brain activity, judgments of response accuracy, and types of errors. J Exp Psychol Hum

678 Percept Perform. 26:141-151. 
679 Shalgi S, Deouell LY. 2012. Is any awareness necessary for an Ne? Front Hum Neurosci.

$680 \quad 6: 1-15$

681 Singmann H, Bolker B, Westfall J, Aust F, Ben-Shachar MS. 2020. Package afex: Analysis

682 of factorial experiments. R package version $0.27-2$.

683 Steinhauser M, Yeung N. 2010. Decision processes in human performance monitoring. J

684 Neurosci. 30:15643-15653.

685 Tibshirani R. 1996. Regression shrinkage and selection via the Lasso. J R Stat Soc Series B

686 Stat Methodol. 58:267-288.

687 Turner BO, Paul EJ, Miller MB, Barbey AK. 2018. Small sample sizes reduce the

688 replicability of task-based fMRI studies. Commun Biol. 1:62.

689 Ullsperger M, Harsay HA, Wessel JR, Ridderinkhof KR. 2010. Conscious perception of

690 errors and its relation to the anterior insula. Brain Struct Funct. 214:629-643.

691 Van Boxtel GJ, Van Der Molen MW, Jennings JR. 2005. Differential involvement of the

692 anterior cingulate cortex in performance monitoring during a stop-signal task. J

693 Psychophysiol. 19:1-10.

694 van Gaal S, Ridderinkhof KR, van den Wildenberg WPM, Lamme VAF. 2009. Dissociating

695 consciousness from inhibitory control: Evidence for unconsciously triggered response

696 inhibition in the stop-signal task J Exp Psychol Hum Percept Perform. 35:1129-1139.

697 van Veen V, Carter CS. 2006. Error detection, correction, and prevention in the brain: A brief

698 review of data and theories. Clin EEG Neurosci. 37:330-335. 
699 Wessel JR. 2012. Error awareness and the error-related negativity: Evaluating the first decade

700 of evidence. Front Hum Neurosci. 6.

701 Wessel JR, Danielmeier C, Ullsperger M. 2011. Error awareness revisited: Accumulation of

702 multimodal evidence from central and autonomic nervous systems. J Cogn Neurosci.

$703 \quad 23: 3021-3036$.

704 Zigmond AS, Snaith RP. 1983. The hospital anxiety and depression scale. Acta Psychiatr

705 Scand. 67:361-370.

706 